\title{
Pseudomyxoma peritonei, a rare tumors of the abdomen: Palliative treatment and response to low-dose capecitabine and/or bevacizumab
}

\author{
Stefanis Aristotelis and Albertsson Maria* \\ Deptartment of Oncology, University Hospital Linköping, Sweden
}

\begin{abstract}
Pseudomyxomaperitonei is a rare abdominal tumor originating from epithelial appendiceal cancer. Its incidence is estimated at 1 per million per year. In this chapter, we present case reports of two separate patients treated with palliative intent with low-dose capecitabine daily \pm bevacizumab and therapy beginning after clear radiological and clinical signs of disease progression. During the period of palliative low-dose capecitabine treatment, both patients had at least a two-year period of stabilized disease and progression-free interval with an outstanding quality of life. Low-dose capecitabine and/or bevacizumab may be beneficial in the palliative treatment of pseudomyxoma peritonei.
\end{abstract}

\section{Introduction}

The literal translation of "pseudomyxoma peritonei" is "false mucinous tumor of the peritoneum". This tumor belongs to a group of unusual abdominal tumors characterized by the production and extensive accumulation of mucus within the abdomen and pelvis. In clinical terms, pseudomyxoma peritonei (PMP) describes a syndrome that produces its symptoms by massive mucus tumor production, resulting in a "jelly belly". The primary site and cause of PMP is the appendix. There are two types of mucin-producing tumors arising from the appendix and leading to PMP, and they display different biology and prognosis [1].

Treatment for PMP has been primary surgical intervention with cytoreductive surgery (CRS) followed by heated intraperitoneal chemotherapy (HIPEC) [2], while systemic chemotherapy treatment has mostly been administered on the basis of clinical experience of individual clinics. There are very few published data about the outcomes, due to the small number of patients.

A study including nearly 2300 patients undergoing cytoreductive surgery showed that this method had a low mortality rate (around $2 \%$ ) but was associated with a high proportion $(24 \%)$ of patients with major complications [3].

Here we report two cases treated with continuous low-dose capecitabine $(500 \mathrm{mg} \times 2$ in case 1 and $500 \mathrm{mg} \times 1$ in case 2$)$ with stable disease for long periods ( $>24$ months) and no side effects.

\section{Case report 1}

A 68-year-old male presented in the Emergency Room in August 2011 with complaints of diffuse abdominal pain, hematemesis, and acute diarrhea. After complete investigation with computed tomography (CT), magnetic resonance tomography, and diagnostic laparoscopy with multiple biopsies, the patient was diagnosed with PMP and referred for CRS and perioperative HIPEC at a specialized center (Figure 1). Surgery was performed 7 months after primary diagnosis, and was not macroscopically radical (R1 resection). Six months after surgery, CT showed recurrent disease (Figure 2), and the patient was once again referred to the specialized center. However, he was not considered suitable for a new HIPEC treatment, and was therefore referred to our oncology department for palliative treatment. Treatment was initiated with capecitabine (500 mg x 2) in May 2013, and the patient was followed on a regular basis with laboratory tests and CT. During two years of treatment there were no signs of tumor progression (Figure 3). The patient had an outstanding quality of life with no or only minor side effects; this was also reflected in laboratory tests (Figure 4). When the first signs of disease progression occurred, 24 months after palliative capecitabine was initiated, bevacizumab was added to the systemic treatment. The treatment is ongoing. The patient continues his oncological therapy and evaluation is scheduled in November 2015, which will mark 3 months after initiation of Avastin while continuing capecitabine daily. His life quality remains excellent.

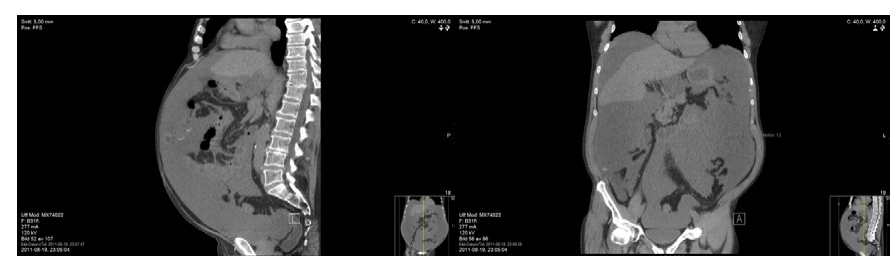

Figure 1a, 1b. Case report 1 .

Correspondence to: Albertsson Maria, Department of Oncology, University Hospital Linköping, Sweden; E-mail: Maria.Albertsson@regionostergotland.se

Key words: pseudomyxoma peritonei, rare abdominal tumor, rare abdominal tumor, bevacizumab

Received: November 14, 2015; Accepted: November 30, 2015; Published: December 04, 2015 


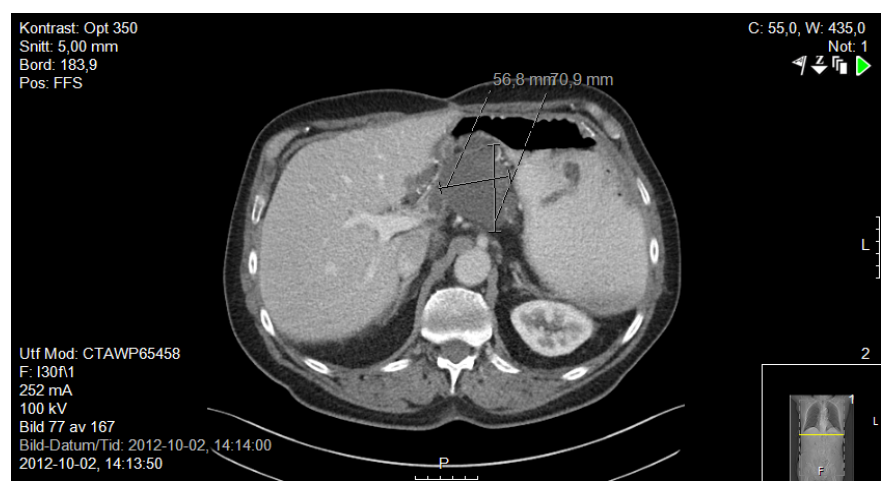

Figure 2a. 6 months after surgery.

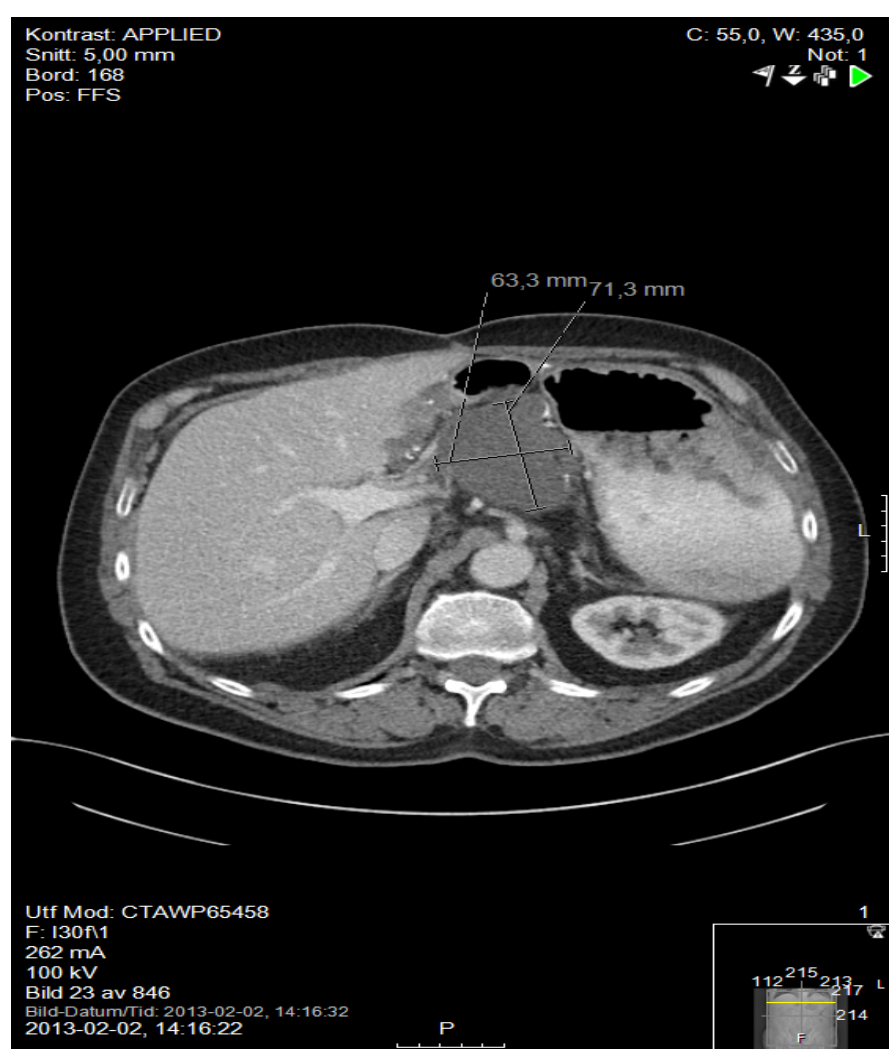

Figure 2b.12 months after surgery and before initiating capecitabine.

\section{Case report 2}

A 63-year-old woman was diagnosed in 2004 with stage III mucin-producing ovarian cancer, and underwent laparotomy with total hysterectomy following six cycles of chemotherapy (paclitaxel/ carboplatin).

Six years later, in December 2010, the patient presented in the Emergency Room with diffuse abdominal pain, high fever, and signs of sepsis. She was treated for likely pyelonephritis with intravenously administered antibiotics. Since her symptoms rapidly abated, she then underwent CT scan imaging of the abdomen, which revealed an abscess in the right side of the pelvis and strong suspicion of peritoneal carcinomatosis. The patient responded to intravenous antibiotics with radiological reduction of the abscess. However, despite several cytology tests of the abscess fluids, no malignant cells could be identified.
Despite all diagnostic efforts and multiple cytology tests, no tumor could be diagnosed, and so an exploratory laparotomy was performed with biopsies from multiple sites including the peritoneum. The results showed low-grade appendiceal mucinous neoplasm/well-differentiated mucinous cystadenocarcinoma of the vermiform appendix. This suggested low-grade PMP, with the appendix tumor being the probable primary tumor site.

Treatment was initiated with doxorubicin/carboplatin, starting 1 month after histological diagnosis and continuing for four cycles. Evaluation with CT showed stable disease. The patient suffered from a

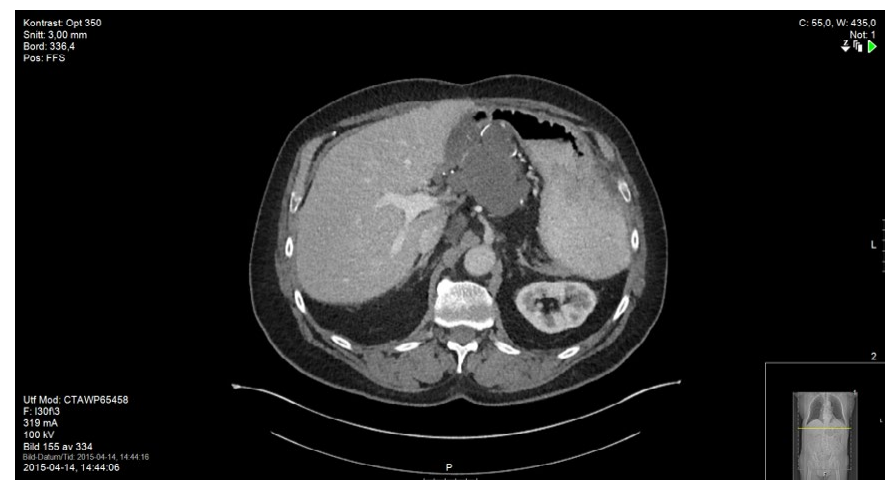

Figure 3. 23 months after initiating daily capecitabine, status quo.

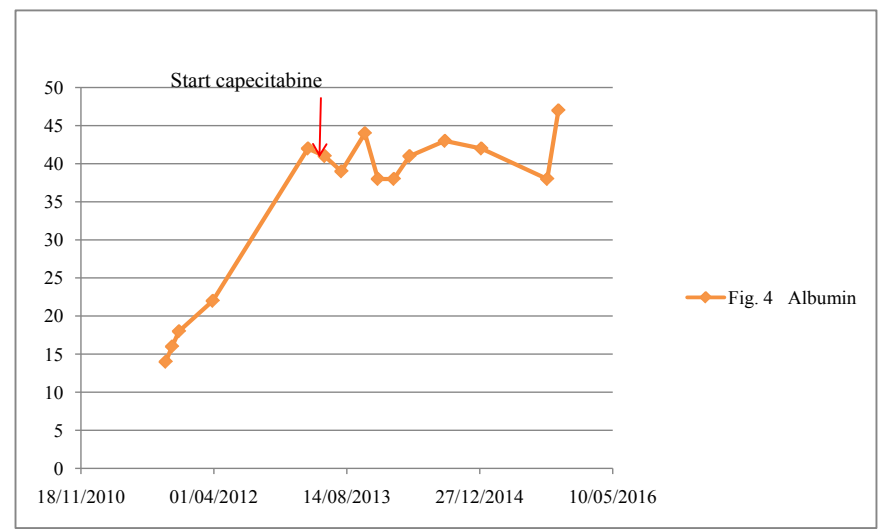

Figure 4a. Albumin.

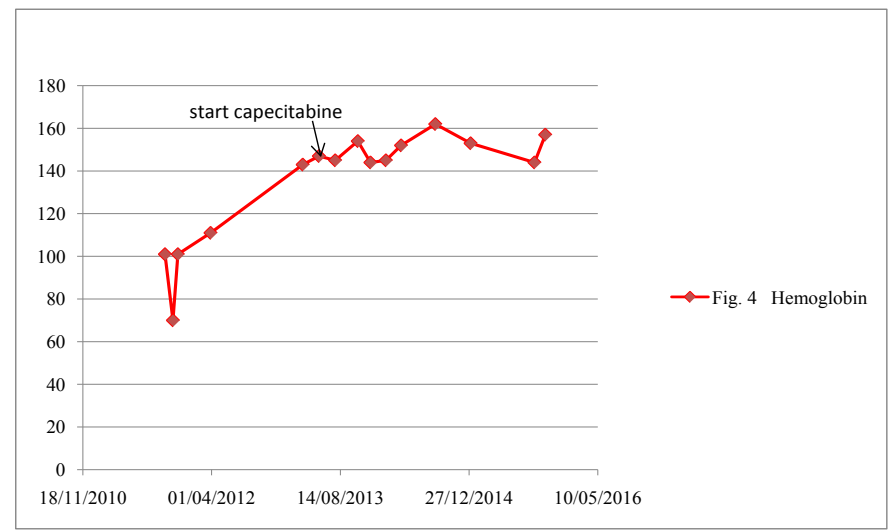

Figure 4b. Hemoglobin. 
myocardial infarction in April 2011, and also experienced side effects from the chemotherapy.

The patient was referred to the gastrointestinal oncology section. She was not considered suitable for intensive chemotherapy, both for tumor biology reasons and because of her heart disease. She was recommended daily low-dose capecitabine $(500 \mathrm{mg} \times 1)$ in October 2011 .

Evaluation with CT scan 3 months after initiation of this treatment showed stable disease. The patient then had a 23-month progressionfree interval as confirmed with regular clinical follow-up with laboratory tests and CT scans. During this period her quality of life was excellent, with no side effects reported at follow-ups. Her major problems during this time were her diabetes, high blood pressure, and finally another myocardial infarction. This diminished the possibilities for further anticancer treatment.

Five months after discontinuation of low-dose systemic treatment, the patient passed away due to deterioration of health and disease progression (Figure 5-9).

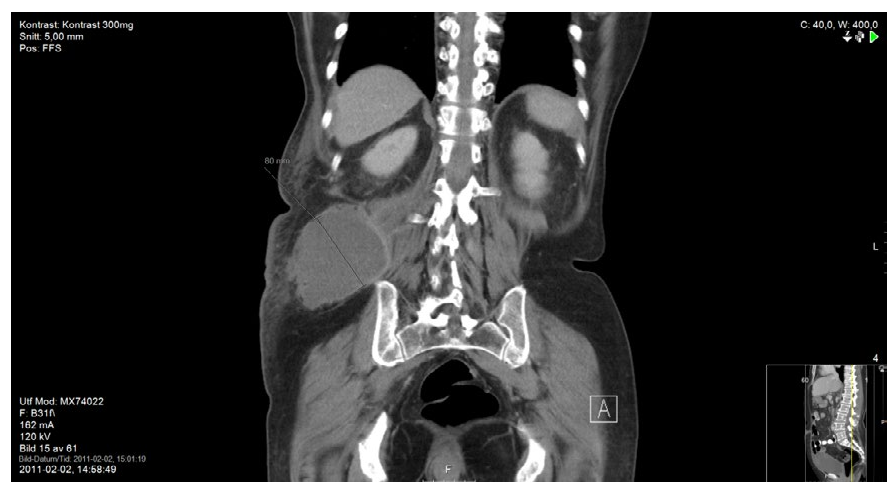

Figure 5a. Prior to diagnosis.

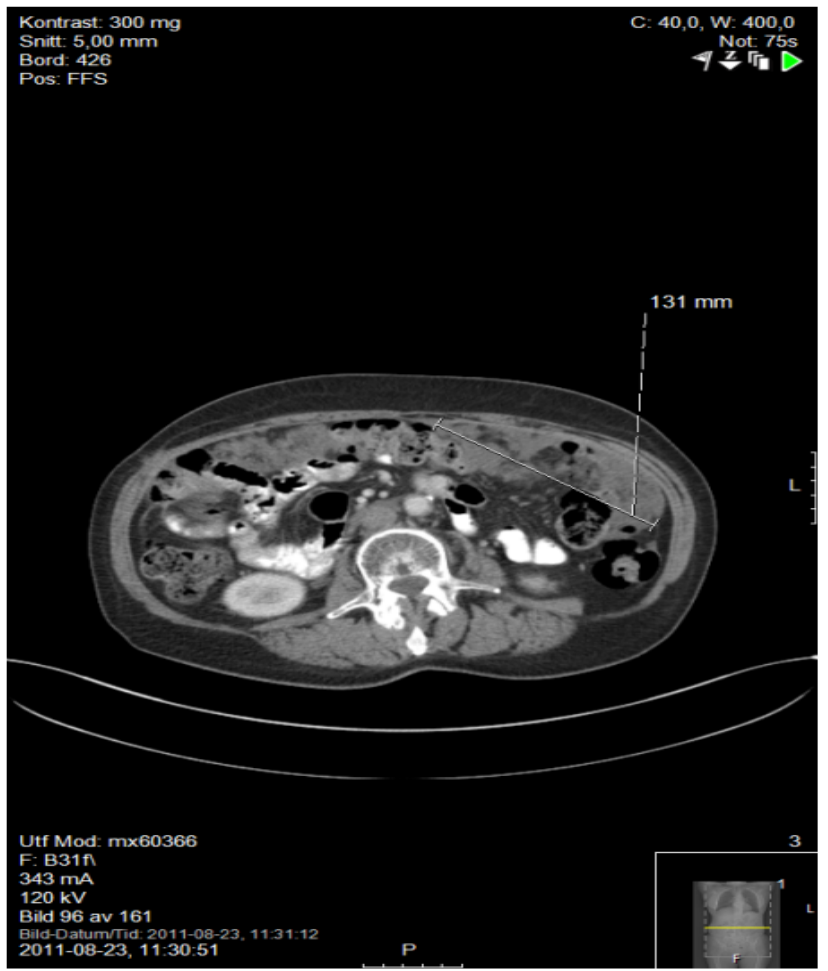

Figure 5b. Before initiating doxorubicin/carboplatin.

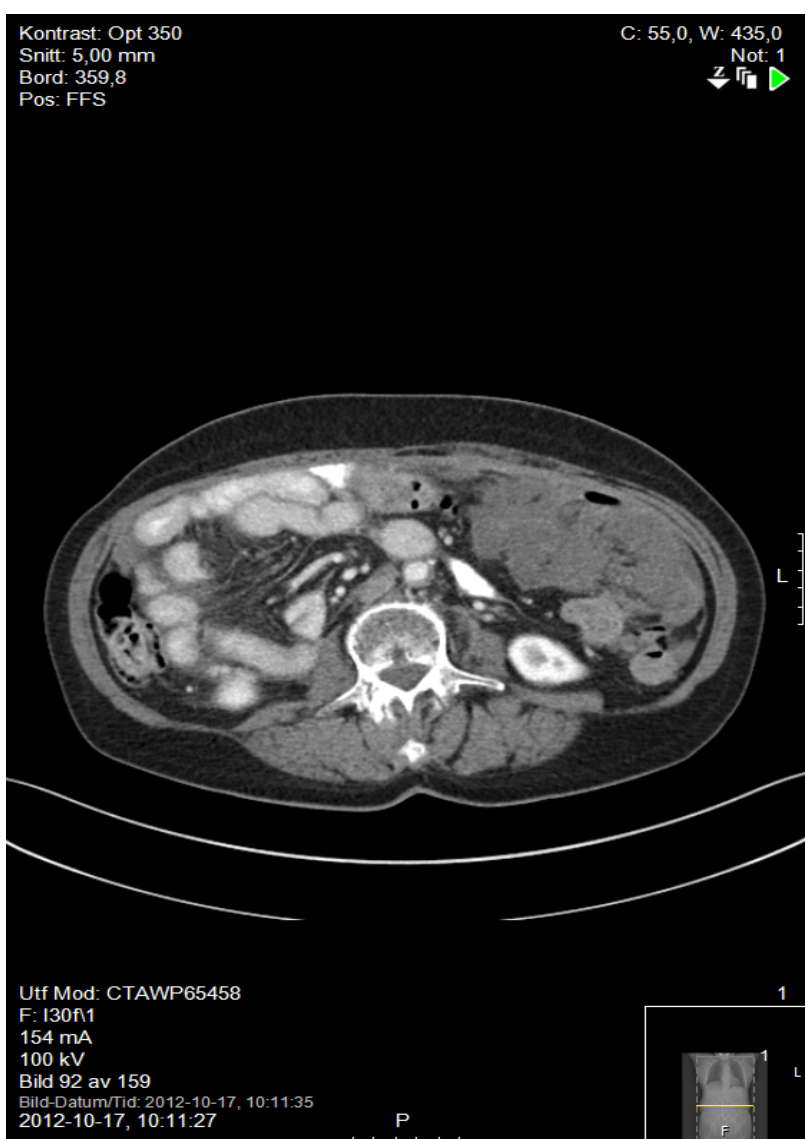

Figure 6. 12 months since start of capecitabine, status quo.

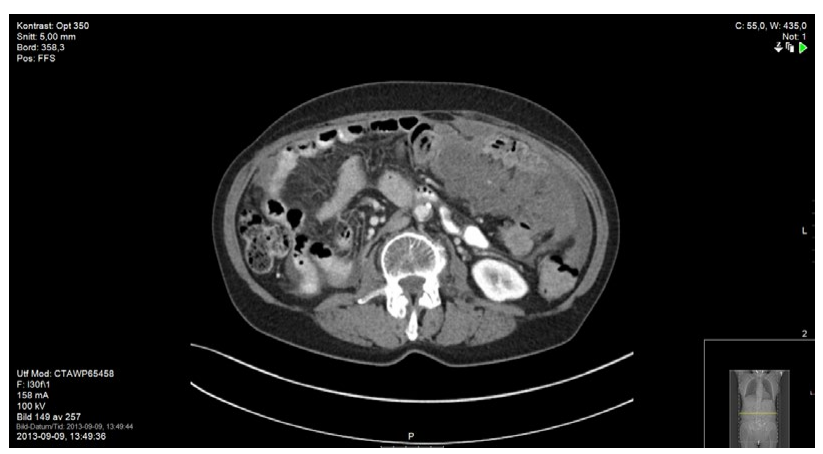

Figure 7a. 23 months since start of capecitabine; CT shows one suspected liver metastasis (Figure 7b), other sites remain status quo.

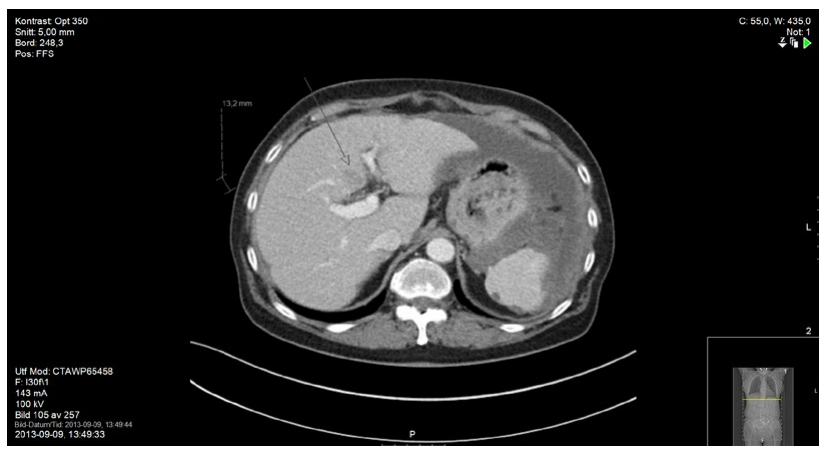

Figure 7b. Suspected liver metastasis. 


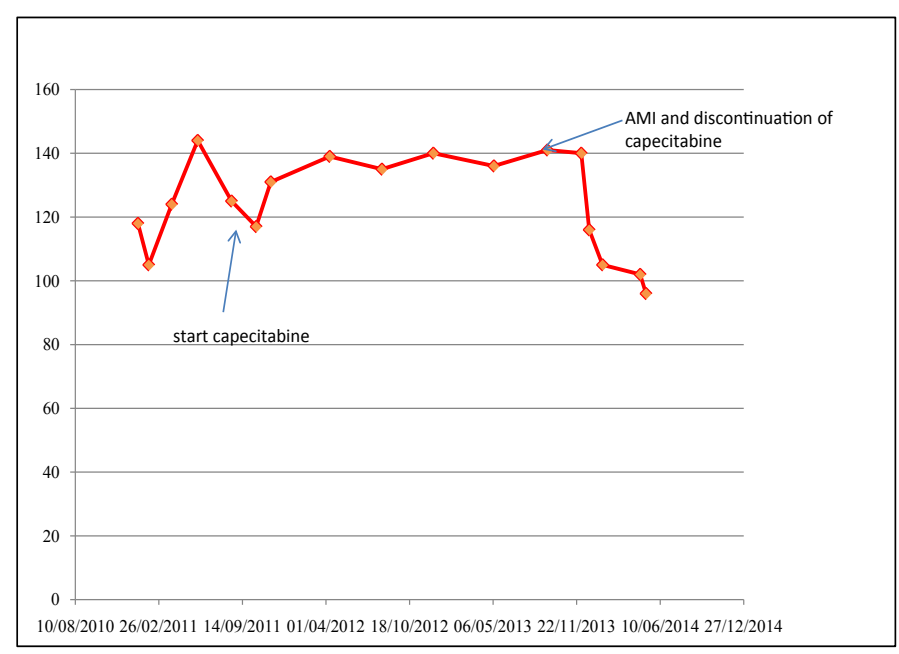

Figure 8. Hemoglobin.

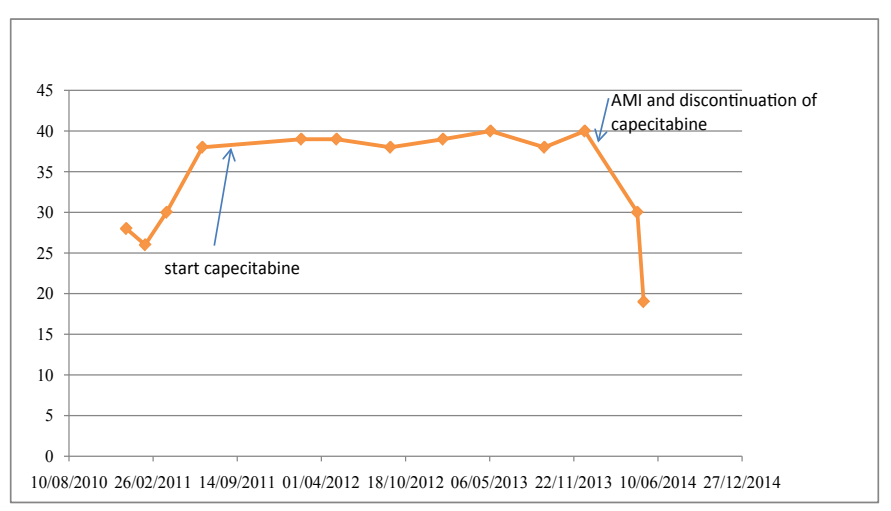

Figure 9. Albumin.

\section{Discussion}

Sugarbaker has proposed that the term "pseudomyxomaperitonei" should be strictly applied to a pathologically and prognostically homogeneous group of cases. These cases are characterized by histologically benign peritoneal tumors that are frequently associated with an appendiceal mucinous adenoma and have a unique natural history. Pathologically, the peritoneal mucinous lesions are termed "disseminated peritoneal adenomucinosis" or simply "adenomucinosis". Cases of peritoneal carcinomatosis, regardless of the presence of abundant extracellular mucin, are excluded from this definition.

Patients with PMP originating from the rupture of a mucinproducing adenoma of the appendix have a more favorable prognosis in comparison with PMP originating from a mucinproducing adenocarcinoma of the appendix. However, the pathologic classification of PMP is still both confusing and controversial [4]. In 1995, Ronnett et al. [5] described three categories: diffuse peritoneal adenomucinosis (DPAM), peritoneal mucinous carcinoma (PMCA), and an intermediate/discordant PMCA subtype (PMCA-I/D).

DPAM is described as a low-grade process with a good prognosis, while PMCA, which is caused by adenocarcinoma of the appendix, is characterized by poor prognosis. PMCA-I/D shows characteristics of both DPAM and PMCA, and has an intermediate prognosis. The importance of better PMP subtyping was made clear by a study of the outcomes for 2298 patients from 16 specialized units who underwent CRS for PMP. Five-year overall survival was $81 \%$ for DPAM, $78 \%$ for PMCA-I/D, and 59\% for PMCA [3].

A retrospective review of treatment of PMP showed that systemic chemotherapy has played only a minor role. This, however, is directly related to the good results that curative surgical intervention has shown over the years. Although the disease incidence is restricted to approximately 1 per million per year, a great number of these patients cannot undergo total CRS because of tumor localization and spreading. Even among patients that undergo total CRS, a large percentage very quickly show signs of recurring disease. At this point, the introduction of palliative treatment is of critical importance. The wellbeing of the patient and control of the disease should be the primary goal, followed by other goals such as treatment costs and outpatient benefits. In this study, we observed progression-free intervals of more than 24 months in two patients with previously progressive disease in the setting of PMP palliation with low-dose capecitabine. These patients had an excellent quality of life with no or only minor side effects. Considering low treatment costs and outpatient therapy that complements tumor control and a good quality of life, low-dose capecitabine shows major benefits in treating palliative patients with PMP.

Our results are in concordance with an earlier report regarding treatment of PMP with capecitabine and bevacizumab, which also showed good results [6-14]. These results make it clear that low-dose capecitabine may be beneficial in the palliative treatment of PMP, and offers an excellent alternative in future treatment of such borderline tumors.

\section{Author's contribution}

SA Drafted the manuscript and made the literature searching. MA designed the study. Both read and approved the final manuscript.

\section{References}

1. Associates S.O (u.d.). [http://www.surgicaloncology.com/pmp.htm]

2. Sugarbaker PH (1995) Peritonectomy procedures. Ann Surg 221: 29-42. [Crossref]

3. Chua TC, Moran BJ, Sugarbaker PH, Levine EA, Glehen O, et al. (2012) Early- and long-term outcome data of patients with pseudomyxoma peritonei from appendiceal origin treated by a strategy of cytoreductive surgery and hyperthermic intraperitoneal chemotherapy. J Clin Oncol 30: 2449-2456. [Crossref]

4. Nakakura EK (2012) Pseudomyxoma peritonei: more questions than answers. J Clin Oncol 30: 2429-2430. [Crossref]

5. Ronnett BM, Zahn CM, Kurman RJ, Kass ME, Sugarbaker PH, et al. (1995) Disseminated peritoneal adenomucinosis and peritoneal mucinous carcinomatosis: a clinicopathologic analysis of 109 cases with emphasis on distinguishing pathologic features, site of origin, prognosis, and relationship to 'pseudomyxomaperitonei'. Am J Surg Pathol 19: 1390-1408. [Crossref]

6. Sun WL, Hutarew G, Gradl J, Gratzl M, Denz H, et al. (2009) Successful antiangiogenic combination therapy for pseudomyxoma peritonei with bevacizumab and capecitabine. Cancer Biol Ther 8: 1459-1462. [Crossref]

7. Smeenk RM, van Velthuysen ML, Verwaal VJ, Zoetmulder FA (2008) Appendiceal neoplasms and pseudomyxoma peritonei: a population based study. Eur J Surg Oncol 34: 196-201. [Crossref]

8. Sugarbaker PH, Chang D (1999) Results of treatment of 385 patients with peritoneal surface spread of appendiceal malignancy. Ann Surg Oncol 6: 727-731. [Crossref]

9. Baratti D, Kusamura S, Nonaka D, Langer M, Andreola S, et al. (2008) Pseudomyxoma peritonei: clinical pathological and biological prognostic factors in patients treated with cytoreductive surgery and hyperthermic intraperitoneal chemotherapy (HIPEC). Ann Surg Oncol 15: 526-534. [Crossref]

10. Sherer DM, Abulafia O, Eliakim R (2001) Pseudomyxoma peritonei: a review of current literature. Gynecol Obstet Invest 51: 73-80. [Crossref] 
11. Ferreira CR, Carvalho JP, Soares FA, Siqueira SA, Carvalho FM (2008) Mucinous ovarian tumors associated with pseudomyxoma peritonei of adenomucinosis type: immunohistochemical evidence that they are secondary tumors. Int $J$ Gynecol Cancer 18: 59-65. [Crossref]

12. Ronnett BM, Yan H, Kurman RJ, Shmookler BM, Wu L, et al. (2001) Patients with pseudomyxoma peritonei associated with disseminated peritoneal adenomucinosis have a significantly more favorable prognosis than patients with peritoneal mucinous carcinomatosis. Cancer 92: 85-91. [Crossref]
13. Glehen O, Mohamed F, Sugarbaker PH (2004) Incomplete cytoreduction in 174 patients with peritoneal carcinomatosis from appendiceal malignancy. Ann Surg 240: 278-285. [Crossref]

14. Sugarbaker PH, Alderman R, Edwards G, Marquardt CE, Gushchin V, et al. (2006) Prospective morbidity and mortality assessment of cytoreductive surgery plus perioperative intraperitoneal chemotherapy to treat peritoneal dissemination of appendiceal mucinous malignancy. Ann Surg Oncol 13: 635-644. [Crossref]

Copyright: $\odot 2015$ Aristotelis S. This is an open-access article distributed under the terms of the Creative Commons Attribution License, which permits unrestricted use, distribution, and reproduction in any medium, provided the original author and source are credited. 